\title{
Experimental search for the electron electric dipole moment with laser cooled francium atoms
}

\author{
T. Inoue - S. Ando · T. Aoki • H. Arikawa - S. Ezure · K. Harada • T. Hayamizu • \\ T. Ishikawa • M. Itoh • K. Kato • H. Kawamura • A. Uchiyama • T. Aoki • \\ K. Asahi • T. Furukawa • A. Hatakeyama • K. Hatanaka • K. Imai • \\ T. Murakami · H. S. Nataraj · T. Sato · Y. Shimizu · T. Wakasa • H. P. Yoshida • \\ A. Yoshimi · Y. Sakemi
}

Published online: 2 December 2014

(C) Springer International Publishing Switzerland 2014

\begin{abstract}
A laser cooled heavy atom is one of the candidates to search for the permanent electric dipole moment (EDM) of the electron due to the enhancement mechanism and its long coherence time. The laser cooled francium (Fr) factory has been constructed to perform the electron EDM search at the Cyclotron and Radioisotope Center, Tohoku University. The present status of Fr production and the EDM measurement system is presented.
\end{abstract}

Proceedings of the 5th Joint International Conference on Hyperfine Interactions and International Symposium on Nuclear Quadrupole Interactions (HFI/NQI 2014) Canberra, Australia, 21-26 September 2014

T. Inoue $(\bowtie)$

Frontier Research Institute of Interdisciplinary Sciences, Tohoku University, 6-3 Aoba, Aramaki, Aoba-ku, Sendai, Miyagi, 980-8578, Japan

e-mail: inoue_t@cyric.tohoku.ac.jp

T. Inoue $\cdot$ S. Ando $\cdot$ T. Aoki $\cdot$ H. Arikawa $\cdot$ S. Ezure $\cdot$ K. Harada $\cdot$ T. Hayamizu $\cdot$ T. Ishikawa

M. Itoh · K. Kato · H. Kawamura · A. Uchiyama · Y. Sakemi

Cyclotron and Radioisotope Center, Tohoku University, Miyagi, Japan

T. Aoki

Graduate School of Arts and Sciences, University of Tokyo, Tokyo, Japan

K. Asahi · T. Sato

Department of Physics, Tokyo Institute of Technology, Tokyo, Japan

T. Furukawa

Department of Physics, Tokyo Metropolitan University, Tokyo, Japan

\author{
A. Hatakeyama \\ Department of Applied Physics, Tokyo University of Agriculture and Technology, Tokyo, Japan \\ K. Hatanaka $\cdot$ H. P. Yoshida \\ Research Center for Nuclear Physics, Osaka University, Osaka, Japan \\ K. Imai \\ Advanced Science Research Center, Japan Atomic Energy Agency, Ibaraki, Japan
}


Keywords CP violation $\cdot$ Electric dipole moment $\cdot$ Laser cooling $\cdot$ Radioactive atom

\title{
1 Introduction
}

Several theoretical models beyond the standard model (SM) have been proposed to solve the problems unanswered in the SM, such as why the present universe is dominated by matter. These models have additional CP violating processes which do not appear in the SM. The permanent electric dipole moment (EDM) is a suitable observable for testing the models beyond the SM, since the EDM is the flavor-diagonal observable violating the time reversal symmetry and hence the CP invariance under the CPT theorem [1].

The EDM search is being performed and planned in various types of particles, such as neutron, diamagnetic atom, paramagnetic atom, molecule and charged particle [2]. In this report, we present an experiment to search for the electron EDM by using the laser cooled francium (Fr) atoms at Cyclotron and Radioisotope Center (CYRIC), Tohoku University.

\section{Electron EDM search with laser cooled Fr atoms}

The electron EDM has been searched for by using an atomic or a molecular beam [3, 4]. So far, the EDM experiment has not been performed by using the laser cooled and trapped atoms. The cooling technique supplies some advantages to the EDM search experiment [5]. Since the laser cooled atoms can be locally confined under a high vacuum environment, both the spin decoherence and the effect of the motional magnetic field $\mathbf{B}_{\text {mot }}=\mathbf{v} \times \mathbf{E}$, where $\mathbf{v}$ and $\mathbf{E}$ are a velocity of the atom and an applied electric field, can be suppressed. In the atomic beam experiment, the spin coherence time is limited, and the effect of $\mathbf{B}_{\text {mot }}$ is one of main sources of systematic errors due to the high velocity beam. We plan to apply the laser cooling technique to the Fr atom, which is the heaviest alkali atom. The electron EDM is enhanced in the heavy atomic system, and the Fr atom has the largest enhancement factor of 895 in the alkali atom [6]. Although Fr has no stable isotope, several Fr isotopes, such as ${ }^{210} \mathrm{Fr}$, have enough life time to perform the EDM experiment.

Our planned procedure for the EDM measurement is as follows. Fr is produced by a nuclear fusion reaction. The produced Fr is transported away from the reaction point as an ion to avoid damages from the radiations, such as neutrons and gamma rays, to the detectors and electrical devices. Then, the Fr ion is neutralized to apply the laser cooling technique.

\author{
T. Murakami \\ Department of Physics, Kyoto University, Kyoto, Japan \\ H. S. Nataraj \\ Indian Institute of Technology Roorkee, Uttarakhand, India \\ Y. Shimizu \\ Department of Physics, Tohoku University, Miyagi, Japan \\ T. Wakasa \\ Department of Physics, Kyushu University, Fukuoka, Japan
}

A. Yoshimi

Research Core for Extreme Quantum World, Okayama University, Okayama, Japan 
The Fr atom is pre-cooled by a magneto-optical trap (MOT). After that, the Fr atom is introduced into an optical lattice and the EDM measurement is performed in the presence of the electric field.

\section{Laser cooled Fr factory}

The laser cooled Fr factory, which is being constructed at CYRIC, consists of a beam swinger magnet, the Fr ion source, the ion transportation system and the MOT system.

Fr is produced through the nuclear fusion reaction with the ${ }^{18} \mathrm{O}^{5+}$ primary beam of 100 $\mathrm{MeV}$ accelerated by an AVF cyclotron and ${ }^{197} \mathrm{Au}$ target as ${ }^{18} \mathrm{O}+{ }^{197} \mathrm{Au} \rightarrow{ }^{215-x} \mathrm{Fr}+x \mathrm{n}$. Since the work function of $\mathrm{Au}(5.1 \mathrm{eV})$ is larger than the ionization potential of $\mathrm{Fr}(4.0 \mathrm{eV})$, the produced Fr is extracted as an ion from the surface of the Au target according to the Saha-Langmuir equation (surface ionization). Since diffusion and a surface condition on the target are important for surface ionization, our Au target can be heated up over the melting point of $\mathrm{Au}$. The liquid has a clean surface compared with the solid, and another advantage is that the extraction time of the Fr ion from the target material is faster than the solid type target due to the diffusion time in the liquid. In fact, the improvement of the extraction efficiency of Fr ions was observed with the target temperature around the Au melting point [7]. A rubidium $(\mathrm{Rb})$ atomic beam can be injected into the Au target in our ion source. Since the $\mathrm{Rb}$ atom has a similar chemical property to the Fr atom, pilot experiments can be performed anytime by using the $\mathrm{Rb}$ beam without the cyclotron operation. Until now, the extraction rate of Fr ions has been achieved to be $10^{6}$ ions/s corresponding to about $40 \%$ extraction efficiency.

The extracted Fr ions are transported about $10 \mathrm{~m}$ away from the reaction point by using one electrostatic deflector, three electrostatic quadrupole triplets and three steerer electrodes. Then, Fr ions are injected into the MOT system. The MOT is employed as a pre-cooling system for the Fr atom. The current MOT system developed is based on the system operated at SUNY [9], which consists of a turnable neutralizer and an octagonallike glass cell, as shown in Fig. 1a. The neutralizer is a thin yttrium (Y) target whose work function $(3.2 \mathrm{eV})$ is lower than the ionization potentials of both Fr and $\mathrm{Rb}(4.2 \mathrm{eV})$. An inner surface of the glass cell is coated with octadecyltrichlorosilane (OTS) to prevent the adsorption of atoms on the surface. The operation procedure of the MOT system is as follows. The transported Fr ions are injected into the $\mathrm{Y}$ target. Then, the $\mathrm{Y}$ target is turned up to the glass cell and typically heated up to $700{ }^{\circ} \mathrm{C}$ in order to release $\mathrm{Fr}$ or $\mathrm{Rb}$ as the neutral atom. Since laser lights and magnetic field gradients, which are required for the MOT, are prepared around the glass cell, the released atoms can be trapped. After the release, the Y target is returned, and the ions are injected into the Y target again. The MOT for the alkali atoms needs the two types of lights; namely, a trapping light and a repumping light, because the atoms have a hyperfine structure. The trapping and repumping lights for the $\mathrm{Rb}$ atom are supplied from two external cavity laser diodes (ECLDs), while the trapping light for the Fr atoms comes from a titanium-sapphire laser and the repumping from an ECLD. An iodine molecule which has rich energy levels was employed to confirm the frequency of the trapping laser light for the Fr MOT, since there is no Fr reference cell due to the absence of a stable Fr isotope. The frequency stabilization of the repumping light for the Fr-MOT was realized by using the offset-locking method [8].

Recently, we tried the Fr-MOT experiment, since we trapped the neutralized Rb atom by using the MOT system as shown in Fig. 1b. However, the fluorescence from the trapped Fr atoms was not observed. Several possible reasons are considered for not observing the 


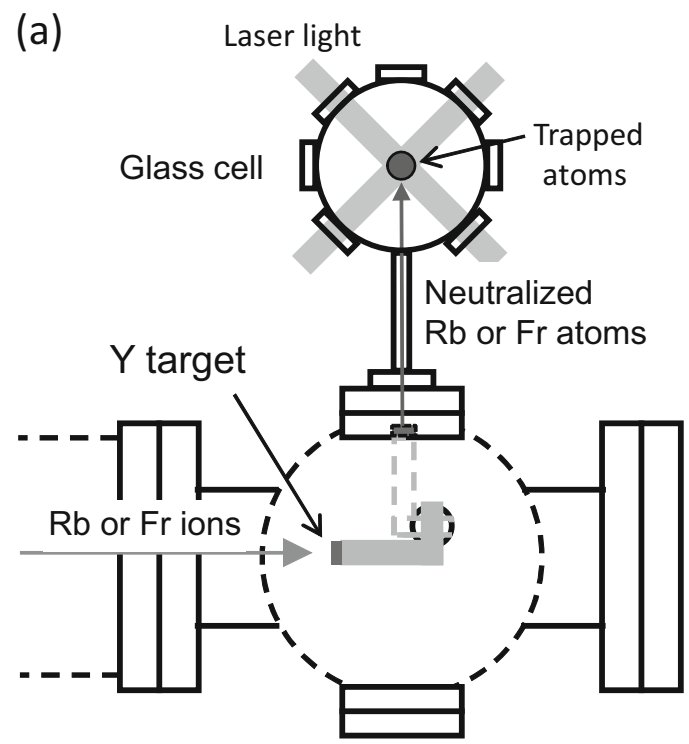

(b)

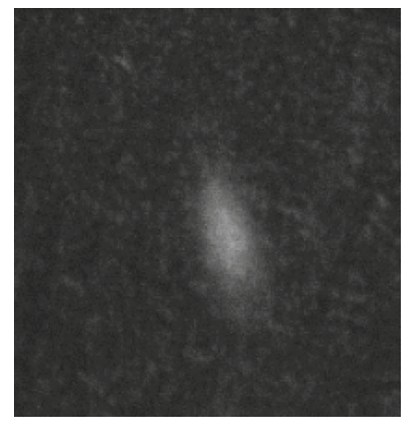

Fig. 1 a MOT system. b Fluorescence form the Rb atoms trapped in the MOT

trapped Fr atoms. For example, the transported ions include many contaminations and the expected number of trapped Fr atoms is extremely small. In order to overcome these conditions, we installed a Wien filter in the Fr beam line, and the observation system for the small number of trapped atoms is being developed.

\section{EDM measurement system}

Toward the EDM measurement, we need to develop the optical trapping system, EDM cell with electrode, magnetometer, magnetic field shielding system etc. At present, these developments are in progress.

Since the EDM is detected as a change of energy difference between the spin sublevels, the EDM measurement requires a spin degree of freedom. However, the atoms trapped in the MOT, which utilizes the interaction between the spin and the magnetic field, have no spin degree of freedom. In order to realize the laser trapping without the magnetic field, as the first step, we are developing an optical dipole trap (ODT) by using the Rb atom. The ODT utilizes the interaction between an electric field of the ODT light and a dipole moment of the atom induced by the ODT light. Until now, we have trapped the Rb atoms of about $2 \times 10^{3}$ in ODT.

A bias magnetic field is applied to define a quantization axis for the atomic spin. The fluctuation of this field produces the false EDM effect $d_{f}$ as $d_{f}=\mu\left(B_{\uparrow \uparrow}-B_{\uparrow \downarrow}\right) / E$, where $B_{\uparrow \uparrow}$ and $B_{\uparrow \downarrow}$ are the bias fields when the electric field is applied parallel and antiparallel to it, respectively. In our assumed experimental conditions, we need to monitor the fluctuation of $B_{\uparrow \uparrow}-B_{\uparrow \downarrow}$ with the precision of less than $10 \mathrm{fT}$ to aim the EDM precision of $10^{-29} \mathrm{ecm}$. In order to realize such precision of magnetic field measurement, the magnetometer based on a nonlinear magneto-optical rotation (NMOR) effect [10] of the Rb atom is under development. With the frequency modulated (FM) light [11], we observed 


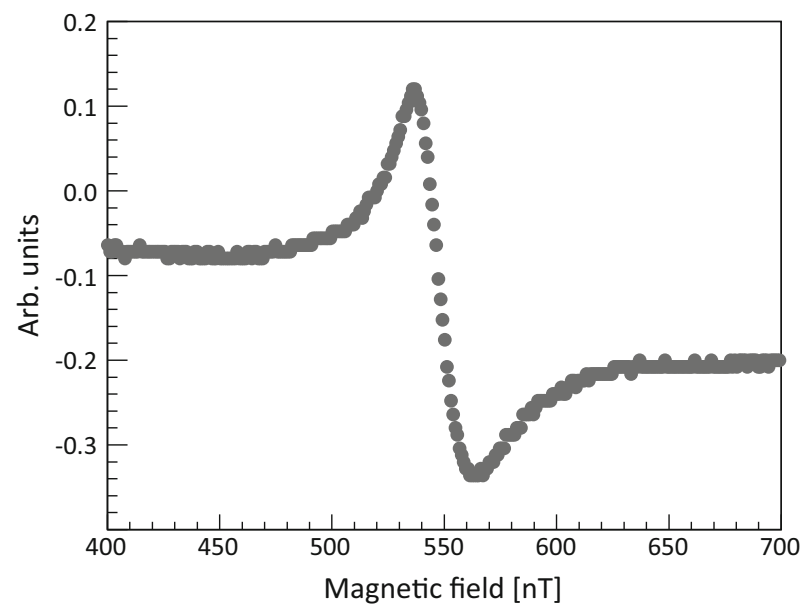

Fig. 2 NMOR spectrum with the FM light. The vertical axis is proportional to a rotation angle of a linear polarized light, which depends on the applied magnetic field. The spectrum is observed in the finite magnetic field region

the NMOR spectrum in the finite range of the magnetic field, which will be applied in the planned EDM experiment, as shown in Fig. 2.

The line width of the NMOR spectrum may be limited by an inhomogeneity of the applied field and the collision with an inner surface of a glass cell which confines the $\mathrm{Rb}$ atoms.

\section{Summary}

The laser cooled Fr factory is being constructed at CYRIC toward the search for the electron EDM. Up to this point, the Fr production and transportation have been achieved. Toward the MOT of the neutralized Fr atoms, some developments are in progress. In addition, we have developed the ODT and the magnetometer based on the NMOR effect. After the completion of these developments, we will begin the EDM measurement.

Acknowledgments This work is supported by MEXT/JSPS KAKENHI Grant Numbers 21104005 and 26220705.

\section{References}

1. Kriplovich, I.B., Lamoreaux, S.K.: CP Violation Without Strangeness. Springer, Heidelberg (1997)

2. Jungmann, K.: Searching for electric dipole moments. Ann. Phys. 525, 550-564 (2013)

3. Regan, B.C., Commins, E.D., Schmidt, C.J., DeMille, D.: New limit on the electron electric dipole moment. Phys. Rev. Lett. 88, 071805 (2002)

4. The ACME Collaboration et al:: Order of magnitude smaller limit on the electric dipole moment of the electron. Science 343, 269 (2014)

5. Chin, C., Leiber, V., Vuletic, V., Kerman, A.J., Chu, S.: Measurement of an electron's electric dipole moment using Cs atoms trapped in optical lattices. Phys. Rev. A 63, 033401 (2001) 
6. Mukherjee, D., Sahoo, B.K., Nataraj, H.S., Das, B.P.: Elativistic coupled cluster (RCC) computation of the electric dipole moment enhancement factor of francium due to the violation of time reversal symmetry. J. Phys. Chem. A 113, 12549 (2009)

7. Kawamura, H. et al.: Search for permanent EDM using laser cooled Fr atoms. Hyperfine Interact 214, 133 (2013)

8. Schunemann, U., Engler, H., Grimm, R., Weidemuller, M., Zielonkowski, M.: Simple scheme for tunable frequency offset locking of two lasers. Rev. Sci. Inst. 70, 242 (1999)

9. Aubin, S., Gomez, E., Gulyuz, K., Orozco, L.A., Sell, J., Sprouse, G.D.: Francium developments at Stony Brook. Nucl. Phys. A 746, 459c (2004)

10. Budker, D., Kimball, D.F., Rochester, S.M., Yashchuk, V.V., Zolotorev, M.: Sensitive magnetometry based on nonlinear magneto-optical rotation. Phys. Rev. A 62, 043403 (2000)

11. Kimball, D.F.J., Jacome, L.R., Guttikonda, S., Bahr, E.J.., Chan, L.F.: Magnetometric sensitivity optimization for nonlinear optical rotation with frequency-modulated light: Rubidium D2 line. J. Appl. Phys. 106, 063113 (2009) 